\title{
CALCIUM-SELECTIVE OPTRODES WITH LASER-INDUCED FLUORESCENCE METHOD
} \author{
SHIN-ICHI WAKIDA ${ }^{*}, \underset{\text { YUJI KAWABATA }}{* *}$, TOTARO IMASAKA ${ }^{* *}$, KUNISHIGE HIGASHI $^{*}$ and
NOBUHIKO ISHIBASHI $^{*}$ \\ *Department of Material Chemistry, Government Industrial Research Institute, Osaka, \\ Midorigaoka 1-8-31, lkeda, Osaka 563, Japan \\ ** Department of Applied Chemistry, Faculty of Engineering, Kyushu University, \\ Hakozaki 6-10-1, Fukuoka 812, Japan
}

\begin{abstract}
New calcium-selective optrodes based on a $\mathrm{Ca}^{2+}$-sensing plasticized PVC membrane and a hydrophobic fluorescent probe were prepared with laser-induced fluorescence method. The $\mathrm{Ca}^{2+}$-selective optrodes showed reversible responses within 30 seconds $(99 \%$ response). The optrodes based on hexadecyl-acridine orange (hexadecyl- $\mathrm{AO}^{+}$) showed linear responses between fluorescent intensity and logarithm of the $\mathrm{Ca}^{2+}$ activity from $10^{-6.5}$ to $10^{-3} \mathrm{M}$ with almost the same potentiometric selectivity.
\end{abstract}

Key words calcium optrode, laser-induced fluorescence method, reversible response, neutral carrier, hydrophobic fluorescent probe

Recently, Ion-selective optrodes based on an ion-sensing plasticized PVC membrane with a neutral carrier and a hydrophobic dye have been proposed[1-6]. Especially, optrodes based on the PVC membrane and a hydrophobic fluorescent probe have a remarkable advantage of their reversible responses[4-6]. In this paper, the sensor characteristics and response mechanism of $\mathrm{Ca}^{2+}$ optrodes with a neutral carrier membrane and a fluorescent dye (dodecyl- $\mathrm{AO}^{+}$or hexadecy-AO $\mathrm{O}^{+}$) were discussed.

\section{EXPERIMENTAL}

Apparatus A schematic diagram of the optrodes with laser-induced fluorescence method is shown in Fig. 1. An NEC GLS3200 argon ion laser $(488 \mathrm{~nm})$ was used as an exciting source. The fluorescence $(520 \mathrm{~nm})$ from $\mathrm{Ca}^{2+}$-selective optrodes was measured with a photomultiplier tube (Hamamatsu, R212) equipped with a monochromator (Jpn. Spectroscopic Co., Ltd., CT-10) in the dark place.

Preparation of calcium-selective optrodes The $\mathrm{Ca}^{2+}$-sensing membrane was prepared by dip-coating the mixture of ETH $1001(20 \mathrm{mg})$, DOS $(143 \mathrm{mg})$, PVC $(70 \mathrm{mg})$ and $\mathrm{Na}^{+} \mathrm{TFPB}^{-}(11.2 \mathrm{mg})$ at the end of the optical fiber (core diameter $0.8 \mathrm{~mm}$, cladding diameter $1 \mathrm{~mm}$ ) and then the membrane was partly ion-exchanged in a $10^{-5} \mathrm{M}$ fluorescent probe (dodecyl- $\mathrm{AO}^{+}$or hexadecyl- $\mathrm{AO}^{+}$) solution. A 0.05 $\mathrm{M}$ Tris. buffer solution ( $\mathrm{pH} 7.0)$ was used for the characterization of the optrodes.

\section{RESULTS and DISCUSSION}

Sensitivity The prepared optrodes showed reversible responses to $\mathrm{Ca}^{2+}$ solutions within 30 seconds $\left(99 \%\right.$ response). The calibration curve of the $\mathrm{Ca}^{2+}$ optrodes with hexadecyl-AO ${ }^{+}$is shown in Fig. 2. Selectivity The selectivity was attempted with the mixed solution method as shown in Fig. 2. It was found that the $\mathrm{Ca}^{2+}$ optrodes have a sensor response similar to the Nicolsky-Eisenman equation.

$$
\mathbf{I}=\mathbf{I}^{\mathrm{O}} \mathbf{S} \times \log \left(\mathbf{a}_{\mathbf{i}}+\operatorname{Kopt}\left(\mathrm{a}_{\mathbf{j}}\right) \mathrm{z}_{\mathrm{i}} / \mathrm{z}_{\mathbf{j}}\right) \text {. }
$$

where $\mathbf{I}$ is the measured fluorescence intensity of the optrode, $I^{O}$ is the constant. Kopt is the optical selectivity coefficient in the presence of an interfering ion $\mathrm{j}$. $\mathrm{S}$ is the slope of the optical sensitivity. The Kopt of $\mathrm{Na}^{+}$and $\mathrm{K}^{+}$were $10^{-4.7}$ and $10^{-4.8}$ respectively, and were almost the same as those of the $\mathrm{Ca}^{2+}$ potentiometric sensor.

Stability The fluorescence intensity from the optrodes with dodecyl- $\mathrm{AO}^{+}$decreased considerably because of leaching of dodecyl-AO+ from the PVC membrane. In order to improve the stability, $\mathrm{Ca}^{2+}$ optrodes with more hydrophobic fluorescent prove, hexadecyl-AO ${ }^{+}$were prepared. This optrodes showed fairly stable response $(-4 \% /$ hour $)$. The sensor stability will be more improved by bonding the fluorescent dye with the PVC membrane covalently. As for reproducibility of the optrode, the relative standard deviation was $3.8 \%$ for 10 measurements in $10^{-3} \mathrm{M}$ solution by correcting drift.

Fluorescent characteristics of hydrophobic fluorescent probe The excitation and emmision spectra of hexadecyl- $\mathrm{AO}^{+}$in the DOS and in the buffer solution are shown in Fig. 3. The fluorescence intensity in $\mathrm{DOS}$ is much larger than that in the buffer solution since hexadecyl- $\mathrm{AO}^{+}$is a hydrophobic probe. 


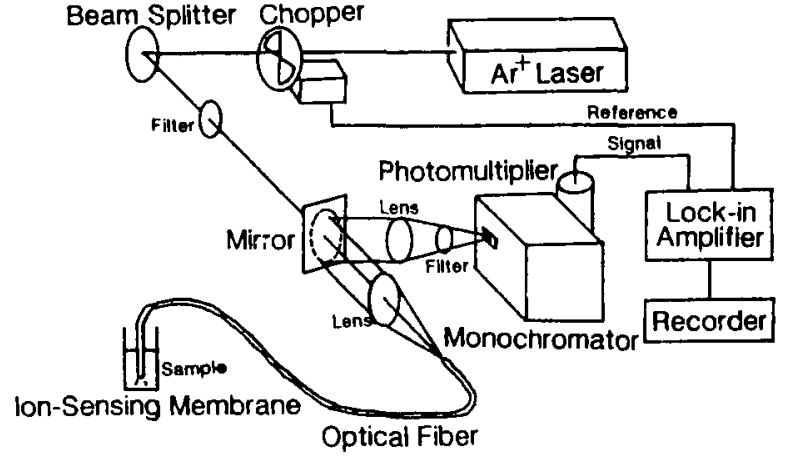

Fig. 1 Schematic diagram of $\mathrm{Ca}^{2+}$-optrodes system.

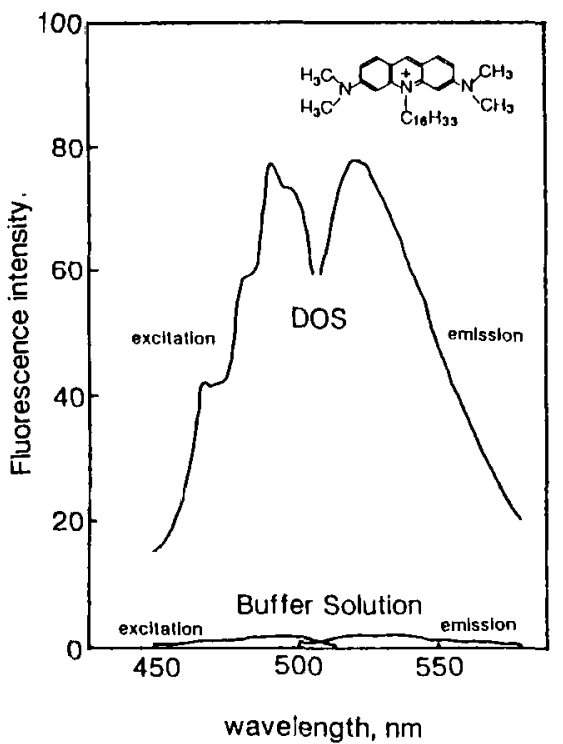

Fig. 3 Excitation and emmision spectra of hexadecyl- $\mathrm{AO}^{+}$in $\mathrm{DOS}$ and buffer solution.

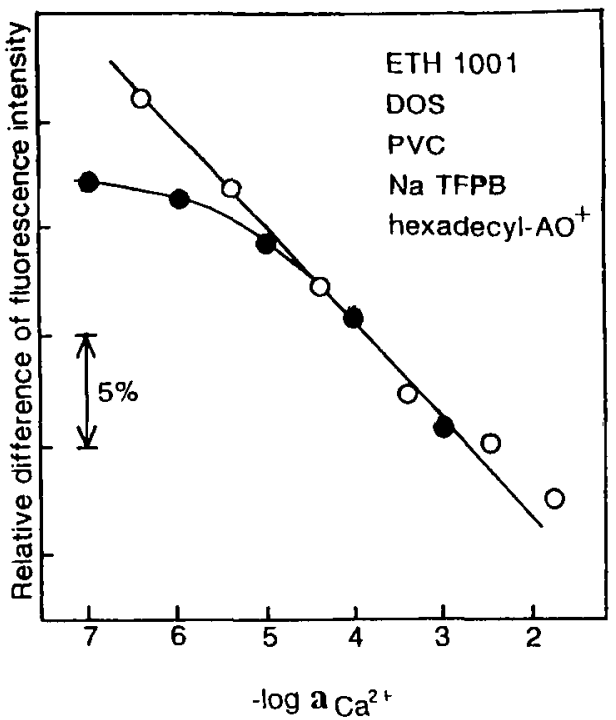

Fig. 2 Calibration curves of $\mathrm{Ca}^{2+}$-optrodes: o standard solution, - with $1 \mathrm{M} \mathrm{KCl}$.
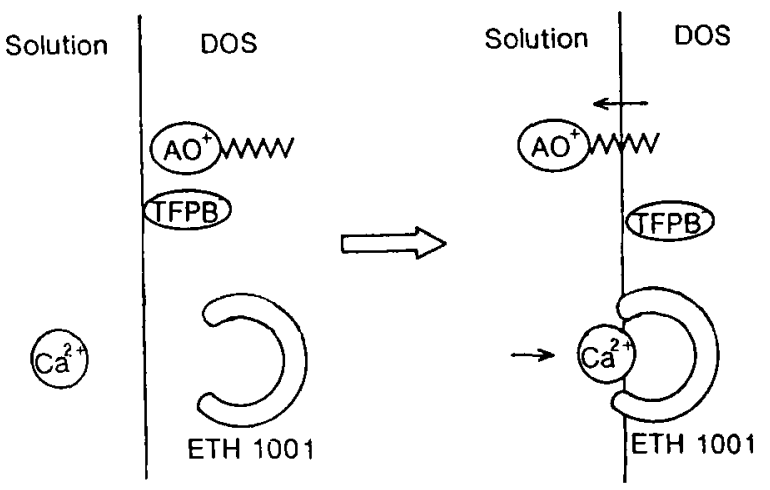

Fig, 4 Proposed response mechanism of optrode.

Response mechanism of optrodes Since the optrodes have relatively rapid and reversible response, it is considered that the response occurs at the interface (probably gel layer) between the buffer solution and the plasticizer (DOS) of the PVC membrane. Moreover, since the optrodes have NicolskyEisenman like responses, it is considered that the response mechanism is similar to that of neutral carrier type ion-selective electrodes. The proposed response mechanism is schematically illustrated in Fig. 4. When the optrodes is soaked in the $\mathrm{Ca}^{2+}$ standard solution of higher concentration, it is considered that while calcium ions may be complexed by the neutral carrier (ETH 1001) in the DOS phase, the charged chromophore of the hydrophobic probe (alkyl acridine orange) may move from DOS to solution phases simultaneously (but a long alkyl chain may remain in the DOS phase) and as a result the fluorescent intensity may decrease with increase of $\mathrm{Ca}^{2+}$ in the solution. When the optrodes is soaked in the lower concentration, the charged chromophore moves from solution to DOS phases in the reverse. However, the investigation on the response mechanism of the optrodes should be accumulated. We are further investigating to clear the response mechanism.

\section{REFERENCES}

1. O. S. Wolfbeis and B. P. H. Schaffar, Anal. Chim. Acta, 198, 1 (1987).

2. W. E. Morf, K. Seiler, B. Rusterholtz and W. Simon, Anal. Chem., 62, 738 (1990).

3. K. Suzuki, H. Ohzora, K. Tohda, K. Miyazaki, K. Watanabe, H. Inoue and T. Shirai, Anal. Chim. Acta, 237, 155 (1990).

4. Y. Kawabata, R. Tahara, T. Kamichika, T. Imasaka and N. Ishibashi, Anal. Chem., 62, 1528 , (1990).

5. Y. Kawabata, T. Kamichika, T. Imasaka and N. Ishibashi, Anal. Chem., 62, 2054 (1990).

6. S. Wakida, Y. Kawabata, T. Imasaka, K. Higashi and N. Ishibashi, Digest of Technical Papers, San Francisco, pp.378-380 (1991). 